\title{
Misinformation effects in an online sample: Results of an experimental study with a five day retention interval
}

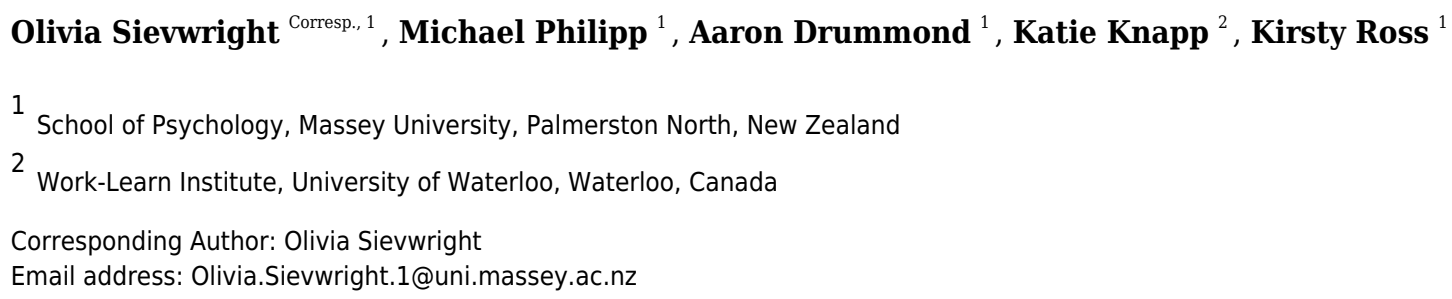

Traditional face-to-face laboratory studies have contributed greatly to our understanding of how misinformation effects develop. However, an area of emerging concern that has been relatively under-researched is the impact of misinformation following exposure to traumatic events that are viewed online. Here we describe a novel method for investigating misinformation effects in an online context. Participants $(\mathrm{N}=99)$ completed the study online. They first watched a 10-minute video of a fictional school shooting. Between five and ten days later, they were randomly assigned to receive misinformation or no misinformation about the video before completing a recognition test. Misinformed participants were less accurate at discriminating between misinformation and true statements than control participants. This effect was most strongly supported by ROC analyses (Cohen's $d=0.59, B F 10=8.34$ ). Misinformation effects can be established in an online experiment using candid violent viral-style video stimuli. 
Olivia M. Sievwright ${ }^{1}$, Michael C. Philipp ${ }^{1}$, Aaron Drummond ${ }^{1}$, Katie Knapp ${ }^{2}$, and Kirsty Ross ${ }^{1}$ 21

${ }^{1}$ School of Psychology, Massey University, Palmerston North, New Zealand

${ }^{2}$ Work-Learn Institute, University of Waterloo, Waterloo, Canada

Corresponding Author:

Olivia Sievwright ${ }^{1}$

School of Psychology, Massey University, Private Bag 11-222, Palmerston North 4442, New Zealand

Email address: Olivia.Sievwright.1@uni.massey.ac.nz

31 
33

34

35

36

37

38

39

40

41

42

43

44

45

46

47

48

49

50

51

52

53

54

55

56

57

58

59

60

61

62

63

64

65

66

67

68

69

70

71

72

\section{Abstract}

Traditional face-to-face laboratory studies have contributed greatly to our understanding of how misinformation effects develop. However, an area of emerging concern that has been relatively under-researched is the impact of misinformation following exposure to traumatic events that are viewed online. Here we describe a novel method for investigating misinformation effects in an online context. Participants $(\mathrm{N}=99)$ completed the study online. They first watched a 10-minute video of a fictional school shooting. Between five and ten days later, they were randomly assigned to receive misinformation or no misinformation about the video before completing a recognition test. Misinformed participants were less accurate at discriminating between misinformation and true statements than control participants. This effect was most strongly supported by ROC analyses (Cohen's d $=0.59, \mathrm{BF} 10=8.34$ ). Misinformation effects can be established in an online experiment using candid violent viral-style video stimuli.

\section{Introduction}

For decades, researchers have demonstrated the harmful effects of misleading post-event information on memory. Numerous laboratory studies have shown that participants exposed to post-event misinformation frequently endorse misinformation as part of their original memory of an event; this is known as the misinformation effect (Loftus, 2005). The application of these findings in legal cases has shed light on the detrimental effects of misinformation in the real world and has contributed to the overturn of wrongful convictions. Misinformation effects may also lead people to remember events as being more traumatic than initially reported (Porter et al., 2008). This study builds on extant misinformation research by testing a novel method for investigating misinformation effects in an online context.

Misinformation effects are traditionally studied in face-to-face laboratory settings using a three-stage procedure (Loftus et al., 1978). First, participants witness a target event, often through a video depicting a crime. Second, after a delay, participants are exposed to misleading information about the target event - this is often presented through a fictional eyewitness account, fictional news report, memory test, or through discussion with a co-witness. Finally, when tested on their memory for the target event (ranging 5 minutes to 2 weeks later), participants frequently report misinformation as part of their original recollection. Misinformation effects have led participants to report seeing a thief using a hammer instead of screwdriver (McCloskey \& Zaragoza, 1985), or even recall non-existent footage of the car crash in which Diana, Princess of Wales was killed (Ost et al., 2002). This procedure has taught us a great deal about how false memories develop in laboratory conditions (for a review see Loftus, 2005).

One area of emerging concern that has been relatively under-researched is the impact of misinformation following exposure to traumatic events that are viewed online. Advancements in Internet technology now offer us immediate access to coverage of real-world traumatic events (Peterson \& Densley, 2017). Consequently, people are inadvertently exposing themselves to footage of such events during their regular Internet use. For example, within 24 hours of the 
73

74

75

76

77

78

79

80

81

82

83

84

85

86

87

2019 Christchurch, New Zealand mosque attacks, the live-stream of the attack had been uploaded to Facebook over 1.5 million times (Besley \& Peters, 2019). This is particularly concerning given accumulating evidence that traumatic content online can have harmful psychological effects for viewers, including post-traumatic stress symptoms, depression, and anxiety (e.g., Pfefferbaum et al., 2019; Redmond et al., 2019; Thompson et al., 2019).

Up-to-the-minute media coverage of unfolding events often includes inaccurate reports based on incomplete information and mistaken details (Rapp \& Salovich, 2018; Rich \& Zaragoza, 2016). For instance, during the Christchurch mosque attacks it was erroneously reported that 'a good guy with a gun' stopped the shooter. In fact the man rushed at the shooter with an electronic payment terminal (Cooke, 2019). Research suggests memory for highly negative events is susceptible to distortion (Nahleen et al., 2020; Strange \& Takarangi, 2012). Exposure to inaccurate information facilitates misinformation effects for real-world traumatic events witnessed through the media. People who view traumatic videos may be vectors of distressing and inaccurate information to the general public. Thus, memory distortions from misinformation effects may have clinical implications even in the absence of legal ones. For instance, even if people who witness violent online videos are unlikely to be called as factual event witnesses in a trial due to the ability to verify facts from the recorded footage, memory distortions from misinformation effects may have implications for psychological distress and, potentially, the development of clinical disorders such as PTSD. Therefore, it is imperative we understand exactly how misinformation effects work in the context of traumatic online media exposure.

Existing misinformation paradigms are not well-suited to investigating misinformation effects in the context of media exposure to traumatic events. Typical misinformation studies use staged videos of a burglary or car crash that clearly depict fictional events. These videos are often not designed to evoke strong emotional responses. Additionally, the artificial laboratory environment may lead to less intense emotional reactions than would typically occur when witnessing a real-life traumatic event (Chae, 2010). Examining misinformation effects in an online context minimises potential experimenter effects and increases ecological validity by exposing people to traumatic media in the context people are most likely to encounter such material—on their own computers.

Two important methodological decisions need to be made when measuring misinformation effects: retention intervals and type of memory test used. Retention intervals are the period between viewing the target event (encoding) and being exposed to misinformation about the event, and the period between exposure to misinformation and memory retrieval. Although longer retention intervals between encoding and misinformation exposure (i.e., at least 24 hours) produce larger misinformation effects (Frost et al., 2002; Paz-Alonso \& Goodman, 2008), most studies use retention intervals of 30 minutes or less. Longer retention intervals between encoding and misinformation exposure have theoretical and pragmatic advantages: theoretically, increasing the retention interval should increase susceptibility to misinformation because event memory decays over time (Conway, 2009; Sekeres et al., 2016). Longer retention 
113 intervals also mimic real-world time intervals between witnessing an event and memory

114 retrieval. An online approach should help to minimise practical issues around longer retention

115 intervals because multiple participant visits to the laboratory are not required.

116 The type of memory test used is important to detecting misinformation effects.

117 Misinformation studies employ different types of memory tests to quantify memory accuracy and

118 misinformation endorsement, including forced-choice recognition, cued recall, free recall,

119 remember/know memory judgements, and source monitoring tests. One of the most common

120 memory tests used in misinformation research is a two-alternative forced-choice recognition test,

121 a test where participants' answers are categorically coded as correct or incorrect. In these tests,

122 participants are asked about some aspect of the target event and are given two possible response

123 options to choose from. For example, participants might be asked to decide whether the thief's t-

124 shirt was blue or green. Although a comprehensive analysis of the advantages and disadvantages

125 of the various memory tests is beyond the scope of the present manuscript, there is debate about

126 what type of test is optimal, particularly in the context of eyewitness evidence, and the primary

127 disadvantage of such categorical measures of memory is that they are relatively insensitive to

128 small shifts in discriminability that fail to reach participants' criteria to change their categorical

129 response (e.g., Wixted \& Mickes, 2018).

130 In this study, we employ a different technique which may be able to detect small

131 discrepancies in people's ability to distinguish between memory for the original event and

132 memory for post-event misinformation. Rather than asking participants to decide between two

133 competing items, we ask participants to make a categorical judgement about how confident they

134 are that a particular item was present in the target video. For example, participants might be

135 asked how certain they are that the thief's t-shirt was blue on a scale from 1 (certain this is false)

136 to 6 (certain this is true). These ratings allow us to plot a receiver operating characteristic (ROC)

137 curve, which provides a clearer indication of precisely how memory accuracy differs at varying

138 confidence levels (Stanislaw \& Todorov, 1999; Wixted \& Mickes, 2018). The advantage of

139 using this type of recognition test is that it may detect subtle changes in memory strength and

140 bias when memory change does not meet the threshold for reporting misinformation as true

141 (Stanislaw \& Todorov, 1999). ROC curves enable us to detect reduced certainty that

142 misinformation is false even when accuracy rates are not explicitly affected. For example,

143 misinforming participants that the thief's t-shirt was blue may cause participants to be less

144 certain that the thief's t-shirt was actually green. Although they may correctly report 'false' for

145 the blue t-shirt, the effect of misinformation can be seen in the reduced certainty ratings.

146 Present Study

147 This study tests the effectiveness of a novel experimental paradigm for investigating

148 misinformation effects in the context of media exposure to trauma using an ostensibly realistic,

149 online, violent viral-style video as the target event, and a one-week retention interval. We also

150 used more sensitive estimates of misinformation effects by measuring participants' confidence in

151 their memory reports to determine discriminability $\left(d^{\prime}\right)$ and points on a ROC curve. To our 
152 knowledge, this is the first study to measure misinformation effects after one week using such a 153 procedure.

154

155

156

157

158

159

160

161

162

163

164

165

166

167

168

169

170

171

172

173

174

175

176

177

178

179

180

181

182

183

184

185

186

187

188

189

190

191

Memory vividness and emotionality ratings have been examined in recent misinformation and trauma therapy studies (e.g., Calvillo \& Emami, 2019; Houben et al., 2018; van Schie \& Leer, 2019). To contribute to future research, a secondary aim of this study was to establish the minimum therapeutically meaningful change in ratings of memory emotionality and vividness across sessions. Although not elaborated on here, the findings from these analyses will help to inform a baseline of memory vividness and emotionality for a future study.

\section{Materials \& Methods}

All procedures reported here involving human participants were in accordance with the ethical standards of the Massey University Human Ethics Committee (Notification number: 4000021787). Participants were informed of the purpose, procedures, and requirements of the study prior to participating. Informed consent was implied through participation in the study and all participants were debriefed as to the purpose of the study at the conclusion of each study. Preregistration information for this study can be accessed at: https://aspredicted.org/ma4st.pdf. The materials and data for this study are openly available on the Open Science Framework (OSF): https://osf.io/r48bm/?view only=8137681d7e044d06a6bef57d4604b32f

\section{Design}

This study consisted of two sessions spaced approximately one week apart (see Figure 1). Session 1 was identical for everyone. In Session 2, half of participants were randomly assigned to the misinformation condition and half were assigned to the no-misinformation control condition. Memory vividness and emotionality ratings were compared across a 2(Condition: misinformation or control) $\times 2$ (Time: session 1 and session 2) mixed factorial design. We carried out an informal pilot test of the study material with a small group of students prior to collecting data. Feedback from the pilot study indicated the study was clear and plausible.

\section{Participants}

Participation was restricted to those who were at least 18 years old. Potential participants were asked not to participate if they had a current or previous diagnosis of post-traumatic stress disorder, depression, or anxiety, or if they had been exposed to a traumatic or violent life event, particularly gun-related violence. We aimed to recruit 100 participants to complete both experimental sessions. This sample size was a compromise between collecting a larger sample size than in previous similar misinformation studies (e.g., Houben et al., 2018) and resource constraints.

One hundred and twenty-nine participants took part in Session 1 of the experiment. Data from 12 participants were excluded for not viewing the entire 10-minute video and data from a further 12 participants were excluded for failing both attention checks. Session 2 was made available to the remaining 105 participants 5-10 days after they completed Session 1. Ninety-nine participants 
192 completed Session 2. No participants expressed suspicion that incorrect details presented in the

193

194

195

196

197

198

199

200

201

202

203

204

205

206

207

208

209

210

211

212

213

214

215

216

217

218

219

220

221

222

223

224

225

226

227

228

229

230

misinformation narrative were intentional or part of the study.

The final sample $(N=99)$ included 53 people ( 33 male, 20 female) in the misinformation condition and 46 (33 male, 11 female, 2 non-binary) in the control condition. The mean age of participants did not differ between the misinformation condition $(M=28.3, \mathrm{SD}=9.32)$ and the control condition $(M=26.6, \mathrm{SD}=8.31), t(97)=0.93, p=0.36$. The mean retention interval between Session 1 and Session 2 also did not differ between the misinformation condition $(M=$ 5.49 days, $\mathrm{SD}=1.15)$ and control condition $(M=5.35$ days, $\mathrm{SD}=1.06), t(97)=0.64, p=0.53$.

\section{Measures/Materials}

Trauma film. In Session 1, participants viewed a 10-minute video comprised of excerpts from the film 'Zero Day' (Coccio, 2002) to temporarily induce mild feelings of distress similar to those experienced in reaction to witnessing a traumatic event. The film depicts a school shooting and the moments leading up to the shooting using ostensible home-video footage and security camera recordings. The video clip begins with two perpetrators introducing themselves to the camera and planning their attack on the school. The perpetrators are then shown entering the school and killing and tormenting multiple students via security camera recordings. The video ends with the perpetrators taking their own lives as an emergency dispatch officer pleads with them over the phone. An image of playing cards was inserted at the end of the video for attention check purposes, described later.

Vividness rating scale. Memory vividness was measured using a Visual Analogue Scale (VAS) during Session 1 and one week later in Session 2. In Session 1, participants rated how vivid (clear) their memory of the trauma film was on a scale from 0 (not vivid at all) to 10 (extremely vivid). At Session 2, participants rated memory vividness using the same scale. They also rated their perceived change in vividness from Session 1 using a 5-point scale with the following response options: (1) a lot more vivid, (2) a little more vivid, (3) the same, (4) a little less vivid, (5) a lot less vivid. We used this to determine the smallest effect size of interest regarding the change in memory vividness over time (Anvari \& Lakens, 2019).

Emotionality rating scale. Memory emotionality was also measured using a VAS in Session 1 and 2. Participants rated how emotional their memory for the trauma film was on a scale from 0 (extremely negative) to 10 (extremely positive). At Session 2, participants rated their perceived change in memory emotionality from Session 1 to Session 2 on a 5-point scale, the same as described above for memory vividness.

Attention checks. Two attention checks were included in Session 1 after participants rated the emotionality and vividness of the video. The first attention check was a directed query; participants were presented with a sliding scale and instructed to leave the question blank and not to click on the scale. The second attention check was a multiple-choice question asking participants what image appeared at the end of the trauma film. Response options included: a cartoon gun, playing cards, schoolbag, or penguin. The correct answer was playing cards. Participants who failed these attention checks were excluded from analyses. 
231

232

233

234

235

236

237

238

239

240

241

242

243

244

245

246

247

248

249

250

251

252

253

254

255

256

257

258

259

260

261

262

263

264

265

266

267

268

269

270

Misinformation manipulation. Misinformation was introduced to participants assigned to the misinformation condition in Session 2 through a 447-word fictional eyewitness narrative describing the events depicted in the traumatic video. The narrative contained 12 true statements about the video (e.g., 'The emergency dispatch lady called out for Andre to pick up the phone') and eight misinformation statements (e.g., 'Andre was in the driver's seat of the car and he was wearing a blue t-shirt' instead of a camouflage shirt). Participants in the control condition did not receive any misinformation, but instead completed a filler-task for five minutes. In the filler-task, participants searched for thirteen words associated with ice cream flavours that were hidden among a $14 \times 14$ grid of letters. Participants dragged the computer mouse over the letters that formed a word to solve the word search puzzle. After five minutes, participants were automatically taken to the next task.

Recognition test. Memory accuracy and susceptibility to misinformation was measured in Session 2 using a true/false recognition test with an associated confidence rating for each test item. The test comprised 24 statements about the traumatic video (e.g., "The blonde perpetrator's name was Cal", "During the shooting Andre tipped over a desk"), with eight statements directly referring to misinformation details presented in the misinformation narrative (e.g., "The darkhaired perpetrator's name was Chris" instead of Andre, "The first gun was retrieved from the backseat of the car" instead of boot). The test also contained 4 false items (foil items) unrelated to information presented in the narrative and 12 true statements_about the video clip. Each statement was presented individually, and participants were not able to skip items or go back to previous items. Participants indicated how certain they were that each statement was either true or false on a 6-point scale ranging from (1) certain this is false, to (6) certain this is true.

Responses of 1-3 for false items were counted as correct and responses of 4-6 for true items were counted as correct (hit). Incorrect answers to misinformation items indicated endorsement of misinformation (false alarm), with a higher number of these questions being incorrect indicating greater susceptibility to misinformation. We measured participants' ability to discriminate between misinformation items and true items using signal detection and receiver operating characteristic curve analyses, described below.

D-prime (d'). We used $d$ ' to measure participants' ability to accurately discriminate between true statements and misinformation statements at test. $d$ ' is derived from the signal detection theory (SDT) of recognition memory, which assumes recognition decisions are based on evidence strength of previously encountered items and new (misinformation or foil) items at test (Stanislaw \& Todorov, 1999). Higher values of $d$ ' indicate a greater ability to accurately discriminate between true statements and misinformation statements. If misinformation effects are present in our study, and misinformation participants are poorer at discriminating between true statements and misinformation statements as hypothesised, $d$ ' should be smaller for the misinformation group than for the control group.

Response bias (c). We used the SDT measure $c$ to compare group differences in response bias. Response bias tells us whether there is a general tendency for participants to respond either "true" or "false" on test items (Stanislaw \& Todorov, 1999). In our study, negative values of $c$ 
271 represent a bias toward responding "true" and positive values of $c$ represent a bias toward

272 responding "false". More negative or positive values of $c$ indicate a stronger bias toward

273 responding "true" or "false", respectively. We expected there would be no difference in overall

274 response bias between the misinformation group and the control group. However, if

275 misinformation effects are present in our study, we would expect the misinformation group to

276 show a greater bias toward responding "true" on misinformation items compared to the control

277 group.

278

279

Receiver operating characteristic (ROC) curves. ROC curves provide a non-parametric and atheoretical estimate of participants' ability to accurately discriminate between true

280

281

282

283

284

285

286

287

288

289

290

291

292

293

294

295

296

297

298

299

300

301

302

303

304

305

306

307

308

309

310 statements and misinformation statements (Stanislaw \& Todorov, 1999). ROC curves are produced by plotting hit rates against false alarm rates for all possible certainty ratings. Chancelevel performance, where the hit rate is equal to the false alarm rate, is represented as a diagonal line. This occurs when participants are unable to discriminate between true items and misinformation items and rely on guessing. Good discrimination accuracy is represented by a curve that bows toward the left. The more the ROC curve bows toward the left, the greater the discrimination accuracy. Based on our hypothesis for misinformation effects, we expect the ROC curve for the control group to bow more toward the left than the ROC curve for the misinformation group. We also expected the curves would show similar hit rates for the misinformation and control groups, but the misinformation group would have higher false alarm rates than the control group.

Area under the curve (AUC). To quantify discrimination accuracy based on the ROC curves, we calculated AUC for the misinformation and control group ROC curves. AUC can be interpreted intuitively as the proportion of times in which participants correctly discriminate true statements from misinformation statements (Stanislaw \& Todorov, 1999). AUC values typically range from 0.5 (chance-level recognition performance) to 1 (perfect recognition performance). Larger AUC indicates greater discrimination accuracy. Consistent with our misinformation effect predictions, we expect the control group will have a larger AUC than the misinformation group, representing poorer discrimination accuracy for the misinformation group.

\section{Procedure}

Participants were recruited via the online participant pool Prolific (www.prolific.co), and the experiment was administered in Qualtrics (www.qualtrics.com). The experiment was advertised as a study of memory for traumatic events involving viewing a 10-minute video of a fictional school shooting and answering some questions about the video. The study was made available for participants to complete on a desktop computer or laptop, and for those whom English is their first language.

Session 1. Participants began by viewing the trauma video. If they did not watch the full video, they were asked to report the timepoint in which they stopped the video. After the video ended, participants rated the emotionality and vividness of the traumatic video and completed the two attention checks. For exploratory purposes, participants were then invited to comment on their experience of viewing the traumatic video. Finally, participants were thanked for their time, 
311 provided contact details for various mental health support services, and reminded they may be 312 asked to participate in another session in 5 days' time. Session 1 took approximately 20 minutes 313 to complete.

314 Session 2. Five days later, participants who completed Session 1 and passed the attention 315 checks were invited to participate in Session 2. Participants were given five days to complete 316 Session 2. They again rated the emotionality and vividness of their memory for the traumatic 317 video and indicated their perceived change in emotionality and vividness from Session 1 to 318 Session 2. Those assigned to the misinformation condition read the misinformation narrative, 319 while those in the control condition completed a filler-task for 5 minutes. Following this, all 320 participants completed a filler-task for a further 5 minutes before completing the recognition test. At the end of the session, participants were thanked for their time, debriefed as to the purpose of the study, and provided with contact details of mental health support services.

\section{Results}

We used the signal detection measure d-prime $\left(d^{\prime}\right)$ and receiver operating characteristic (ROC) curves to assess participants' ability to correctly distinguish between true statements and misinformation statements one week after viewing the traumatic video. We excluded nonmisinformation false items from the analysis so we could compare true items directly to misinformation items. A flattening constant was applied to account for floor and ceiling effects for hit rates and false alarm rates. Standard calculations were used for $d^{\prime}$, response bias (c), and ROC (Stanislaw \& Todorov, 1999). We report 95\% confidence intervals for Cohen's $d$ effect size for each comparison. Post-hoc Bayes' factors for independent samples t-tests with a default Cauchy prior $(0, .707)$ were used to determine the relative evidence in favour of the null or alternative hypothesis for each analysis. Where the assumption of equal variances was violated for between-group comparisons, a Mann-Whitney $U$ test $(U)$ was used.

Results comparing discriminability $\left(d^{\prime}\right)$ for the misinformation and control groups showed a significant difference in correct discrimination between the misinformation $(M=0.21$, $\mathrm{SD}=0.56)$ and control group $(M=0.49, \mathrm{SD}=0.75), t(97)=-2.10, p=.04$, Cohen's $d=0.42$, $95 \% \mathrm{CI}[0.02,0.82]$. These results suggest exposure to misinformation one week after viewing a traumatic video interfered with participants' ability to correctly distinguish between true and false statements at test. However, we found contradictory results for the post-hoc $\mathrm{BF}_{10}=1.46$; indicating only anecdotal evidence of this effect. We calculated $c$ and AUC to see whether we could further clarify the effect of misinformation on discrimination accuracy.

Group comparisons for $c$ showed participants in the misinformation group $(M=0.79, \mathrm{SD}$ $=0.28$ ) were slightly more biased towards responding "true" compared to control participants $(M$ $=0.55, \mathrm{SD}=0.35), U=699, p<.001, d=0.76,95 \% \mathrm{CI}[0.35,1.17]$. The moderate-to-large effect size, and post-hoc $\mathrm{BF}_{10}=91.4$ indicates very strong evidence of the effect of misinformation on a bias towards responding "true". There are two possible explanations for this: first, the bias towards responding "true" may reflect misinformation effects; that is, misinformation group participants responded "true" to a greater proportion of misinformation 
351

352

353

354

355

356

357

358

359

360

361

362

363

364

365

366

367

368

369

370

371

372

373

374

375

376

377

378

379

380

381

382

383

384

385

386

387

388

389

390

items thereby inflating overall response bias. Second, exposure to misinformation after one week may have weakened participants' memory for the original event, leading them to agree with statements and respond "true" more often than disagreeing and responding "false" across all test items. We further examined response bias using ROC analyses.

In addition to signal detection measures, we used area under the ROC curve as a more sensitive measure of discriminability. We plotted hit rates against false alarm rates for both groups at each level of certainty (from "certain this is false" through to "certain this is true"). Figure 2 displays the ROC curves for the misinformation and control groups. The ROC curves further support the signal detection analyses above, with the control group demonstrating a greater bow to the left, moving further away from chance-level performance, and indicating superior discriminability compared to the misinformation group. The ROC curves also show similar hit rates at each certainty level for the misinformation and control groups. However, the misinformation group show higher false alarm rates than the control group. This suggests the bias toward responding "true" for the misinformation group can more likely be explained by a greater tendency for misinformation participants to respond "true" to misinformation statements than control participants. If it were the case that the misinformation group had an overall tendency to respond "true" across all test items, we would expect to see a greater effect on hit rates.

We calculated AUC for the misinformation and control group ROC curves using the inverse of the trapezoidal rule for AUC. This involved dividing the area above the curves into a series of trapezoids and summing the area of each trapezoid. We subtracted this sum from 1 to give the AUC. An independent samples $t$-test comparing AUC revealed a significant difference between groups in discrimination accuracy. Participants in the control group correctly determined whether a statement was true or false $77 \%(\mathrm{SD}=0.12)$ of the time when true statements and misinformation statements were presented during the recognition test. However, participants in the misinformation group correctly identified true and false items $70 \%$ (SD $=$ 0.11 ) of the time, $t(97)=-2.91, p=.004, d=0.59,95 \%$ CI $[0.18,0.99]$. We found a post-hoc $\mathrm{BF}_{10}=8.34$, indicating the data is 8.34 times more likely under the alternative hypothesis (i.e., that participants in the misinformation condition are worse at discriminating between true and false statements) than the null. Although $d^{\prime}$ results showed some ambiguity, our AUC comparisons show a clear effect of misinformation on participants' discrimination accuracy after one week.

\section{Exploratory Analyses}

The analyses in this section were not pre-registered and are therefore exploratory. To explore whether differences in discriminability were due to endorsement of misinformation, we compared hit rates and false alarm rates for both groups. If differences can be attributed to the effects of misinformation, we would expect the misinformation group to have a higher false alarm rate than the control group, but no difference in hit rates between groups. Figure 3 displays the group comparisons of hit and false alarm rates. We found no significant difference in hit rates between the misinformation and control groups, $U=1146, p=.60$. However, the false alarm rate 
391

392

393

394

395

396

397

398

399

400

401

402

403

404

405

406

407

408

409

410

411

412

413

414

415

416

417

418

419

420

421

422

423

424

425

426

427

428

429

430

was significantly higher for the misinformation group than the control group, $U=659, p<.001$, $d=0.91$. Post-hoc $\mathrm{BF}_{10}=1052$ suggests very strong evidence that between-group differences in discriminability between true and false statements can be attributed to inflated false alarm rates for participants exposed to misinformation.

\section{Emotionality and Vividness Ratings}

We used a global transition method (Anvari \& Lakens, 2019) to determine the smallest effect size of interest for changes in memory emotionality and vividness for the trauma video from Session 1 to Session 2. We conducted these analyses to determine a cut-off point for the smallest subjectively detectable change in memory vividness and emotionality that is therapeutically meaningful. Emotionality ratings were missing from 3 participants (2 misinformation, 1 control) and vividness ratings were missing from 1 participant in the misinformation group. Data from 96 participants were analysed for emotionality and 98 participants for vividness. Session 1 and Session 2 emotionality ratings were reverse-coded so that higher ratings represented more negative emotionality.

Figure 4 shows the change in emotionality ratings from Session 1 to Session 2 for the two groups. A mixed-model ANOVA established no significant main effect of time, $F(1,94)=0.84$, $p=.36, \eta^{2}=0.003$, or condition, $F(1,94)=0.67, p=.42, \eta^{2}=0.005$. There was also no significant interaction between time and condition for participants' ratings of emotionality, $F(1,94)=0.19, p=.67, \eta^{2}=0.001$. This suggests participants' memory for the school shooting video was equally negative one week after viewing the video as it was immediately after viewing the video.

To determine the minimum therapeutically meaningful change in memory emotionality from Session 1 to Session 2, we grouped participants' responses into three categories: those reporting "no change" $(n=71)$, "a little change" $(n=23)$, or "a lot of change" $(n=3)$. We focused on participants who reported "a little more positive" or "a little more negative" emotionality at session 2 . These ratings were combined to form the "little change" category. We conducted a Chi-Square test of independence to examine differences between conditions in the number of participants subjectively reporting "no change", "a little change", or "a lot of change" in memory emotionality. No significant differences were found between groups, suggesting no effect of misinformation conditions on perceived change in memory emotionality, $\chi^{2}(2, N=97)$ $=0.81, p=.67$.

A paired-samples t-test found that, for participants who reported "a little change" in emotionality from Session $1(M=6.57, \mathrm{SD}=3.04)$ to Session $2(M=6.24, \mathrm{SD}=1.51)$, there was a non-significant mean decrease in emotionality of $0.33, t(20)=0.50, p=.63, d=0.12,95 \%$ CI $[-0.32,0.54]$. This suggests even for those reporting a subjective sense of change, memory emotionality remained stable over time. As a result, we were unable to calculate the smallest therapeutically meaningful effect for emotionality; however, we were able to establish a clear baseline for memory emotionality for the traumatic video over a one-week period.

Changes in ratings of memory vividness from Session 1 to Session 2 for the misinformation and control groups are displayed in Figure 5. We found a main effect of time, 
431 indicating an overall decrease in vividness for memory of the school shooting video from

432 Session 1 to Session 2, irrespective of experimental condition, $F(1,96)=126.53, p<.001, \eta^{2}=$

433 0.27. There was also a main effect of condition; participants in the misinformation group tended

434 to rate their memory for the school shooting video as more vivid than participants in the control

435 group $F(1,96)=4.79, p=.03, \eta^{2}=0.03$. However, the time $\times$ condition interaction was non-

436 significant, $F(1,96)=0.82, p=.37, \eta^{2}=0.002$.

437 Again, we grouped participants' responses into three categories: those reporting "no 438 change" $(n=26)$, "a little change" $(n=58)$, or "a lot of change" $(n=15)$ in memory vividness. A

439 Chi-Square test of independence examined differences between conditions in the number of 440 participants reporting "no change", "little change", or "a lot of change" in memory vividness 441 from Session 1 to Session 2. No significant differences were found between the misinformation 442 and control conditions, $\chi^{2}(2, N=99)=1.77, p=.41$. We focused on participants who reported 443 their memory was "a little more vivid" or "a little less vivid" at Session 2. A paired-samples t444 test found that, for participants who reported "little change" in memory vividness from Session 1 $445(M=8.10, \mathrm{SD}=1.28)$ to Session $2(M=6.26, \mathrm{SD}=1.15)$, there was a mean decrease in 446 vividness of 1.84 over time, $t(57)=9.87, p<.001, d=1.30$. Memory vividness decreased 447 significantly more for the "little change" group than for the "no change" group, $t(82)=-2.19, p=$ $448.03, d=0.52$. This suggests effect sizes of at least $d=1.30$ represent a therapeutically 449 meaningful change in memory vividness over time, with smaller effect sizes representing 450 changes that are too small to be subjectively perceived.

451

\section{Discussion}

To our knowledge, this is the first study to test misinformation effects for a violent viralstyle video following a one-week retention interval using an online platform. We found only anecdotal evidence that misinformation impaired discrimination accuracy when using $d$ '.

However, using area under the ROC curve, we established moderate evidence that participants who received misinformation were less accurate when discriminating between true statements and misinformation statements than those who received no misinformation. Our results are consistent with other studies finding an effect of post-event misinformation on memory accuracy and misinformation endorsement (e.g., Loftus et al., 1989; Takarangi et al., 2006).

Our findings highlight the added value of ROC analyses for detecting subtle changes in memory strength, even when misinformation may be below the acceptance threshold. Moreover, the medium effect sizes we found are particularly notable given that our study was conducted online where the capacity for monitoring and control is often much lower than in traditional laboratory experiments.

This study extends our understanding of misinformation effects by using novel stimuli, procedures, and context. We elicited misinformation effects outside of a laboratory using a wholly online experiment and realistic target event to simulate the violent viral-style videos that people may come across online. Many participants commented on the realism of our video, likening it to the 2019 Christchurch mosque shootings and the 1999 Columbine High School 
471 massacre. Using an online format, misinformation effects were elicited from a diverse sample

472 that may be more representative of the general population. Moreover, we showed the robustness 473 of misinformation effects under relatively extreme experimental conditions-involving reduced 474 experimental control, a high degree of participant autonomy and trust, and a one-week retention 475 interval.

476

477

478

479

480

481

482

483

484

485

486

487

488

489

490

491

492

493

494

495

496

497

498

499

500

501

502

503

504

505

506

507

508

509

510

We also used more sensitive estimates of misinformation effects by measuring participants' confidence in their memory reports and using this to determine discriminability $\left(d^{\prime}\right)$ and points on a ROC curve. Signal detection has been used in many previous misinformation studies (e.g., Chan et al., 2017; Paz-Alonso et al., 2013; van Bergen et al., 2010), however ROC curves are rarely employed in such research. Some argue that a key advantage is that ROC analyses are unaffected by the assumptions about recognition memory that underlie $d$ ' (Wixted $\&$ Mickes, 2018). Although the direction of effects in our study was consistent across analyses, ROC analyses provided stronger evidence of misinformation effects. This is because ROC curves enabled us to detect instances where misinformation reduced participants' certainty in a recognition judgement, even when they did not overtly accept the misinformation. Future research should consider employing ROC analyses as an additional measure to allow for detection of subtle changes in memory strength in misinformation effect research.

We also explored participants' ratings of vividness and emotionality for their memory of the traumatic video, both immediately after viewing and one week later. Irrespective of exposure to misinformation, participants rated their memory for the video as highly vivid and negative. Our results are consistent with other trauma film paradigm studies which have found that experimental analogues to traumatic events can produce intrusive and distressing memories (James et al., 2016). Our findings also contribute to research on the psychological effects of media exposure to traumatic events; although participants in our study were aware the traumatic video was fictional, they appear to have rated their emotions and the vividness of their memories as relatively intense. This provides some indication of the impact of media exposure to real-life traumatic events, such as the live-streamed footage of the Christchurch attacks, which is likely to be even greater.

Interestingly, participants rated their memory as being equally negative one week after initially viewing the video but experienced a small decrease in memory vividness over the oneweek period. Our results are partially consistent with findings from similar studies, which have found reductions in memory vividness over time (Houben et al., 2018; van Schie \& Leer, 2019). However, these studies also found memory emotionality became more positive over time, which we were unable to replicate. A possible explanation for this is that our video was more distressing than the staged car crash video used in previous studies, thereby we were able to produce more enduring emotional reactions.

Memory vividness tended to be higher for misinformed participants than control participants - across both sessions. According to prominent false memory and recognition decision theories (e.g., fuzzy-trace theory, source monitoring framework, and ballistic accumulator models), poorer memory vividness increases susceptibility to misinformation 
511 (Brainerd \& Reyna, 2002; Brown \& Heathcote, 2008; Johnson et al., 1993). Based on this 512 assumption and our vividness results, we would expect the misinformation group to have better

513 memory performance than the control group. However, we found the opposite; despite

514 perceiving their memory as more vivid, the misinformation group had poorer discrimination

515 accuracy than the control group. Our findings suggest memory vividness did not interfere with

516 misinformation effects and demonstrate that misinformation effects can still occur for memories

517 that are experienced as being relatively vivid.

518 One limitation of this novel misinformation paradigm is that we cannot control for

519 extraneous variables as well in the online setting compared to a laboratory environment.

520 Distraction during the encoding or testing phases may have affected some participants'

521 performance on the recognition test. We attempted to minimise such effects by including

522 multiple attention checks throughout the experiment. A second limitation of this study may be

523 the objectively short interval of five minutes between exposure to misinformation and memory

524 retrieval; however, the implications of this are largely unknown. Further studies should

525 investigate whether the effect of misinformation on retrieval is affected by this interval.

526 Additionally, although participants may have incorrectly responded to recognition test items

527 based on their memory for the narrative, rather than the video, we believe this is unlikely for two

528 reasons. First, we provided participants with clear instructions to answer the questions based on

529 their memory for the video. Second, were participants responding based upon the narrative, we

530 would have expected to see a much larger increase in acceptance of the misinformation items

531 than the moderate effects we observed. Lastly, it is also possible that some participants searched

532 YouTube for the target video between sessions or took notes during initial viewing since we told

533 participants they would be asked questions about the video. However, this seems unlikely in our

534 study, given that no participants achieved recognition accuracy greater than $91.7 \%$ at test.

535 Furthermore, the fact we still found significant effects in our study highlights the robustness of

536 misinformation effects.

537

538 Conclusions

539 This study tested the viability of a novel method for investigating misinformation effects in an

540 online context. To our knowledge, this is the first study to investigate misinformation effects

541 after 1 week using a fully online sample with ostensibly realistic, viral-style, traumatic stimuli.

542 Using signal detection and area under the receiver operating characteristic curve, results showed

543 participants exposed to misinformation one week after viewing a traumatic video were

544 significantly worse at discriminating between true information and misinformation compared to

545 participants not exposed to misinformation. Our results suggest misinformation effects can be

546 established in an online experiment using candid violent viral-style video stimuli. This study also

547 provides evidence for the validity of ROC analyses in misinformation research. We hope our

548 novel materials can further contribute to understandings of memory, misinformation effects, and

549 media exposure to trauma at a faster pace and with more diverse samples than previously

550 achieved. 
551

552

553

554

555

556

557

558

559

Acknowledgements

560 We wish to thank Malcolm Louden for technical assistance in the design of this study.

561

562

563

564

565

566 


\section{References}

568 Anvari F, Lakens D. 2019. Using anchor-based methods to determine the smallest effect size of interest.

569

570

571

572

573

574

575

576

577

578

579

580

581

582

583

584

585

586

587

588

589

590

591

592

593

594

595

596

597

598

599

600

601

602

603

604

605

606

607

608

PsyArXiv. DOI: https://doi.org/10.31234/osf.io/syp5a.

Besley T, Peters MA. 2019. Terrorism, trauma, tolerance: Bearing witness to white supremacist attack on Muslims in Christchurch, New Zealand. Educational Philosophy and Theory:1-11. DOI: 10.1080/00131857.2019.1602891.

Brainerd C, Reyna VF. 2002. Fuzzy-trace theory and false memory. Current Directions in Psychological Science 11:164-169.

Brown SD, Heathcote A. 2008. The simplest complete model of choice response time: Linear ballistic accumulation. Cognitive Psychology 57:153-178. DOI: 10.1016/j.cogpsych.2007.12.002.

Calvillo DP, Emami AS. 2019. Do lateral eye movements increase susceptibility to misinformation? A registered replication. Psychonomic Bulletin \& Review. DOI: 10.3758/s13423-019-01641-6.

Chae Y. 2010. Application of laboratory research on eyewitness testimony. Journal of Forensic Psychology Practice 10:252-261. DOI: 10.1080/15228930903550608.

Coccio B. 2002. Zero Day. Avatar Films.

Conway MA. 2009. Episodic memories. Neuropsychologia 47:2305-2313. DOI: 10.1016/j.neuropsychologia.2009.02.003.

Cooke H. 2019. Christchurch terror attack: Mistruths and conspiracy theories. Stuff.

Forgas JP, Laham SM, Vargas PT. 2005. Mood effects on eyewitness memory: Affective influences on susceptibility to misinformation. Journal of Experimental Social Psychology 41:574-588. DOI: 10.1016/j.jesp.2004.11.005.

Frost P, Ingraham M, Wilson B. 2002. Why misinformation is more likely to be recognised over time: A source monitoring account. Memory 10:179-185.

Horry R, Colton LM, Williamson P. 2014. Confidence-accuracy resolution in the misinformation paradigm is influenced by the availability of source cues. Acta Psychologica 151:164-173. DOI: 10.1016/j.actpsy.2014.06.006.

Houben STL, Otgaar H, Roelofs J, Merckelbach H. 2018. Lateral eye movements increase false memory rates. Clinical Psychological Science 6:610-616. DOI: 10.1177/2167702618757658.

James EL, Lau-Zhu A, Clark IA, Visser RM, Hagenaars MA, Holmes EA. 2016. The trauma film paradigm as an experimental psychopathology model of psychological trauma: intrusive memories and beyond. Clinical Psychology Review 47:106-142. DOI: 10.1016/j.cpr.2016.04.010.

Johnson MK, Hashtroudi S, Lindsay DS. 1993. Source monitoring. Psychological Bulletin 114:3-28.

Loftus EF. 2005. Planting misinformation in the human mind: A 30-year investigation of the malleability of memory. Learning \& Memory 12:361-366. DOI: 10.1101/1m.94705.

Loftus EF, Donders K, Hoffman HG, Schooler JW. 1989. Creating new memories that are quickly accessed and confidently held. Memory \& Cognition 17:607-616. DOI: 10.3758/BF03197083.

Loftus EF, Miller DG, Burns HJ. 1978. Semantic integration of verbal information into a visual memory. Journal of Experimental Psychology: Human Learning and Memory 4:19-31. DOI: 10.1037/0278-7393.4.1.19.

McCloskey M, Zaragoza M. 1985. Misleading postevent information and memory for events: Arguments and evidence against memory impairment hypotheses. Journal of Experimental Psychology: General 114:1-16. DOI: 10.1037//0096-3445.114.1.1.

Peer] reviewing PDF | (2021:02:58274:1:0:NEW 5 Aug 2021) 
609 Nahleen, S., Strange, D., \& Takarangi, M. K. T. (2020). Does emotional or repeated misinformation

610

611

612

613

614

615

616

617

618

619

620

621

622

623

624

625

626

627

628

629

630

631

632

633

634

635

636

637

638

639

640

641

642

643

644

645

646

647

648

649

650

651 increase memory distortion for a trauma analogue event? Psychological Research. https://doi.org/10.1007/s00426-020-01409-x.

Ost J, Vrij A, Costall A, Bull R. 2002. Crashing memories and reality monitoring: Distinguishing between perceptions, imaginations and 'false memories.' Applied Cognitive Psychology 16:125134. DOI: DOI: 10.1002/acp.779.

Paz-Alonso PM, Goodman GS. 2008. Trauma and memory: Effects of post-event misinformation, retrieval order, and retention interval. Memory 16:58-75. DOI: https://doi.org/10.1080/09658210701363146.

Peterson J, Densley J. 2017. Cyber violence: What do we know and where do we go from here? Aggression and Violent Behavior 34:193-200. DOI: 10.1016/j.avb.2017.01.012.

Pfefferbaum B, Nitiéma P, Newman E. 2019. Is viewing mass trauma television coverage associated with trauma reactions in adults and youth? A meta-analytic review. Journal of Traumatic Stress 32:175-185. DOI: 10.1002/jts.22391.

Porter, S., Taylor, K., \& ten Brinke, L. (2008). Memory for media: Investigation of false memories for negatively and positively charged public events. Memory, 16(6), 658-666. https://doi.org/10.1080/09658210802154626

Rapp DN, Salovich NA. 2018. Can't we just disregard fake news? The consequences of exposure to inaccurate information. Policy Insights from the Behavioral and Brain Sciences 5:232-239. DOI: $10.1177 / 2372732218785193$.

Redmond S, Jones NM, Holman EA, Silver RC. 2019. Who watches an ISIS beheading - and why. American Psychologist Advance online publication. DOI: http://dx.doi.org/10.1037/amp0000438.

Rich PR, Zaragoza MS. 2016. The continued influence of implied and explicitly stated misinformation in news reports. Journal of experimental psychology. Learning, memory, and cognition 42:62-74. DOI: $10.1037 / \mathrm{xlm} 0000155$.

van Schie K, Leer A. 2019. Lateral eye movements do not increase false-memory rates: A failed directreplication study. Clinical Psychological Science 7:1159-1167. DOI: $10.1177 / 2167702619859335$.

Sekeres MJ, Bonasia K, St-Laurent M, Pishdadian S, Winocur G, Grady C, Moscovitch M. 2016. Recovering and preventing loss of detailed memory: differential rates of forgetting for detail types in episodic memory. Learning \& Memory 23:72-82. DOI: 10.1101/1m.039057.115.

Stanislaw H, Todorov N. 1999. Calculation of signal detection theory measures. Behavior Research Methods, Instruments, \& Computers 31:137-149. DOI: 10.3758/BF03207704.

Strange, D., \& Takarangi, M. K. T. (2012). False memories for missing aspects of traumatic events. Acta Psychologica, 141(3), 322-326. https://doi.org/10.1016/j.actpsy.2012.08.005

Takarangi MKT, Parker S, Garry M. 2006. Modernising the misinformation effect: the development of a new stimulus set. Applied Cognitive Psychology 20:583-590. DOI: 10.1002/acp.1209.

Thompson RR, Jones NM, Holman EA, Silver RC. 2019. Media exposure to mass violence events can fuel a cycle of distress. Science Advances 5:eaav3502. DOI: 10.1126/sciadv.aav3502.

Wixted JT, Mickes L. 2018. Theoretical vs. empirical discriminability: the application of ROC methods to eyewitness identification. Cognitive Research: Principles and Implications 3:9. DOI: 10.1186/s41235-018-0093-8. 
Figure 1

Schematic representation of the experimental procedure for Session 1 and Session 2.

Session 1

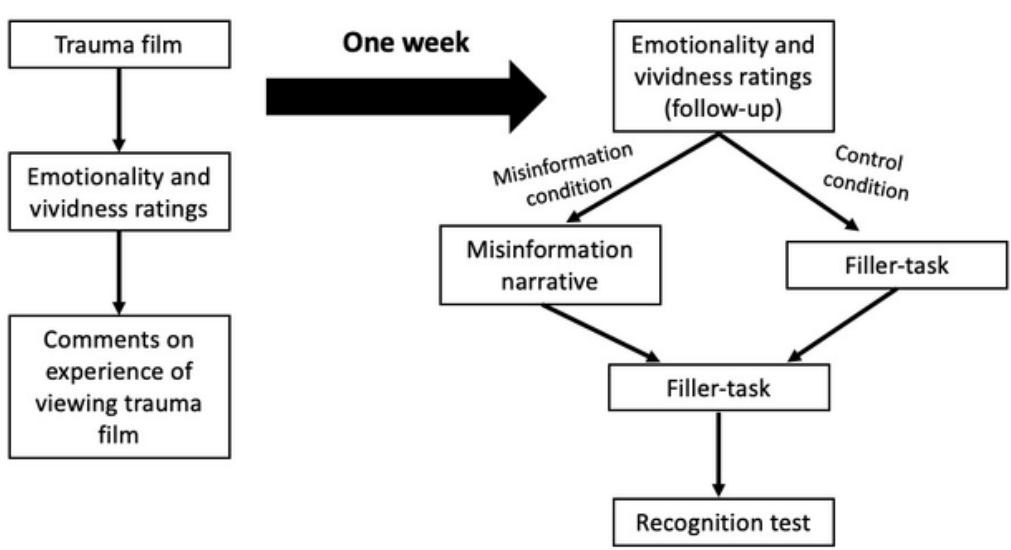


Figure 2

A comparison of receiver operating characteristic $(R O C)$ curves plotting hit rates against false alarm rates between misinformation and control conditions.

The grey dotted line denotes chance performance, where the hit rate is equivalent to the false alarm rate. Each data point along the curve represents a different certainty level for recognition responses, ranging from "certain this is false" to "certain this is true". 


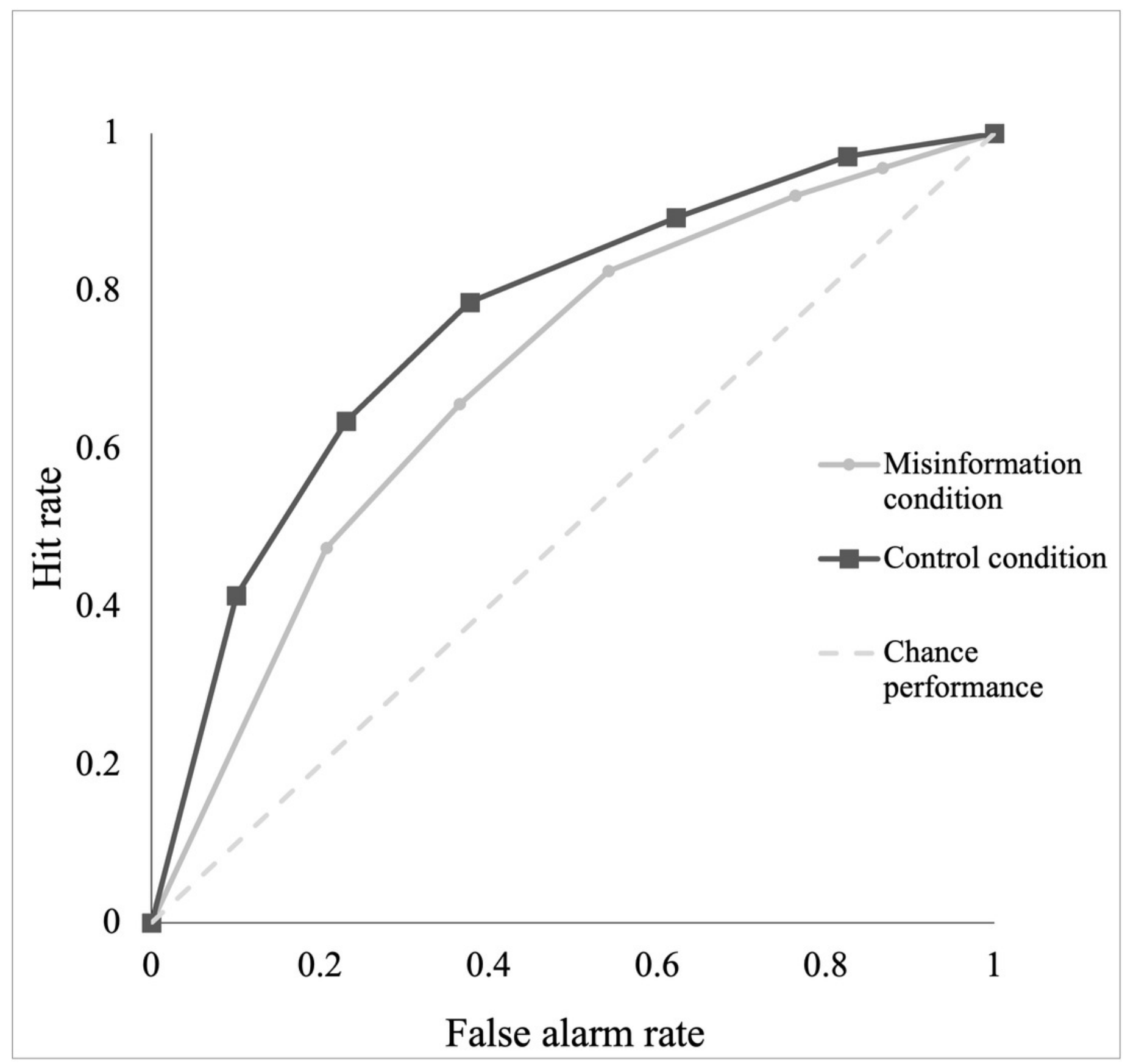




\section{Figure 3}

Recognition test hit and false alarm rates for the misinformation group and control group.

Error bars demonstrate standard error of the mean (SEM) for hit and false alarm rates.

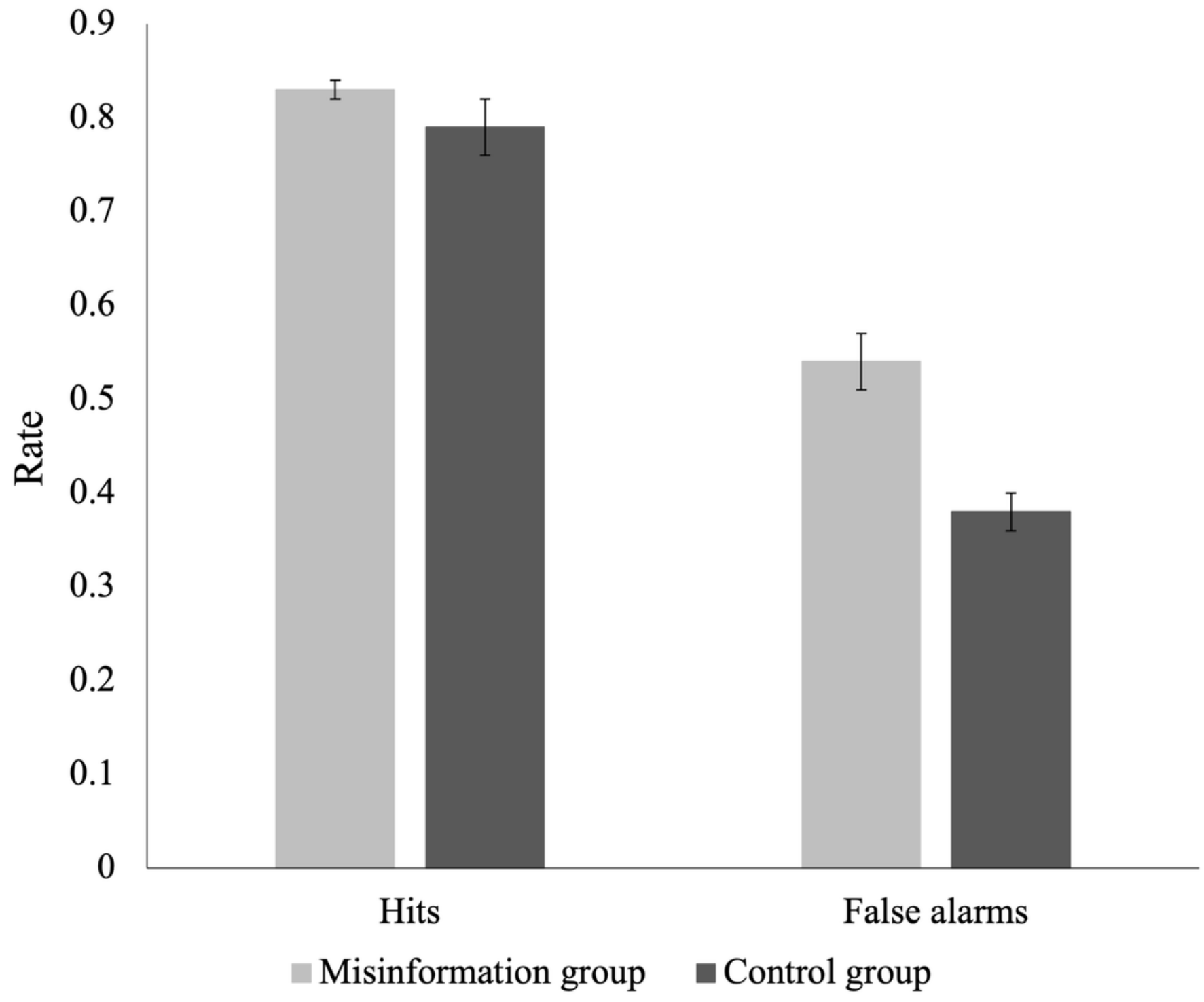


Figure 4

Change in emotionality ratings for the traumatic video from Session 1 to Session 2 for the misinformation and control groups.

Error bars demonstrate standard error of the mean (SEM) for ratings of memory emotionality.

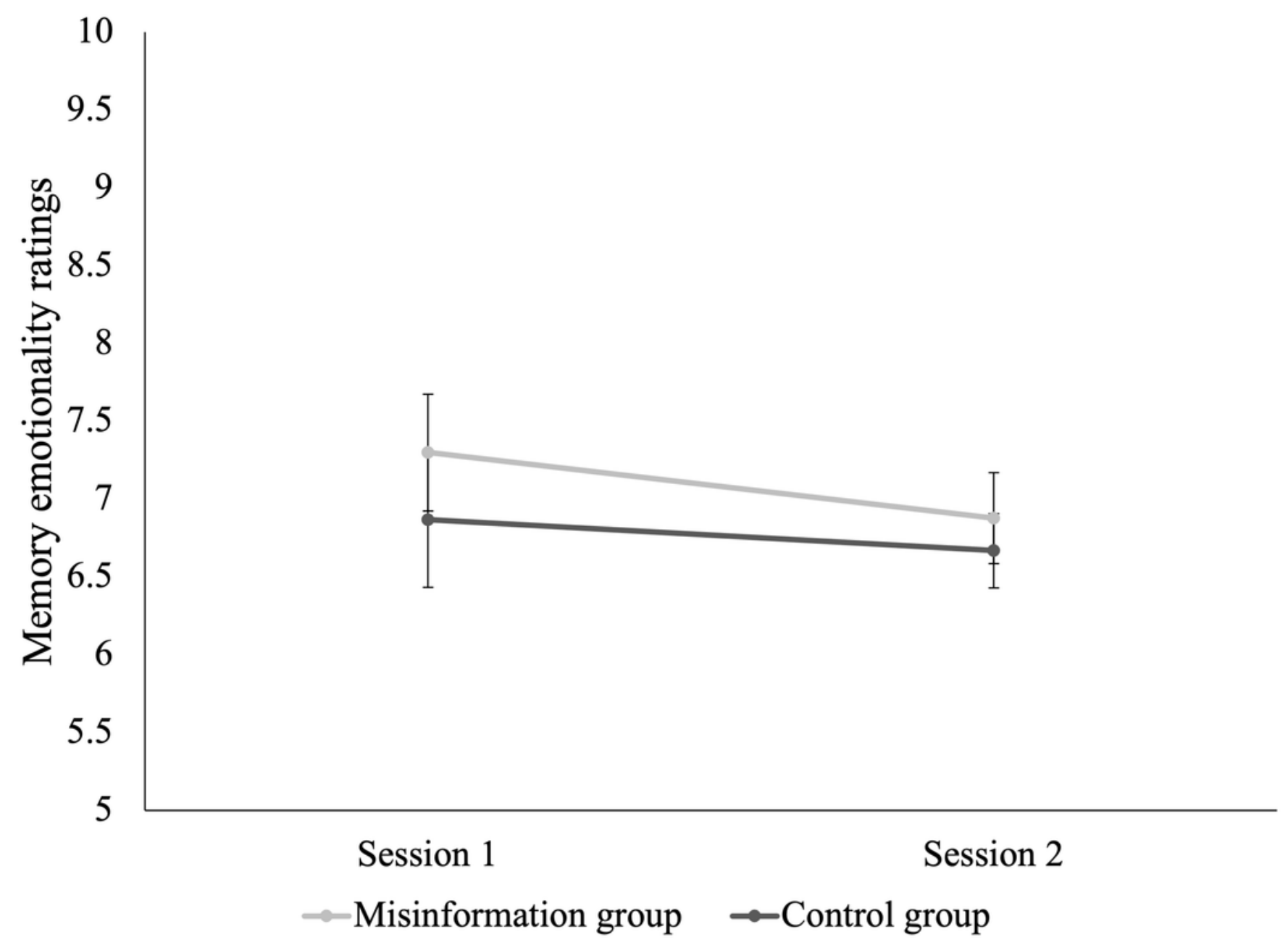


Figure 5

Change in vividness ratings for the traumatic video from Session 1 to Session 2 for the misinformation and control groups.

Error bars demonstrate standard error of the mean (SEM) for ratings of memory vividness.

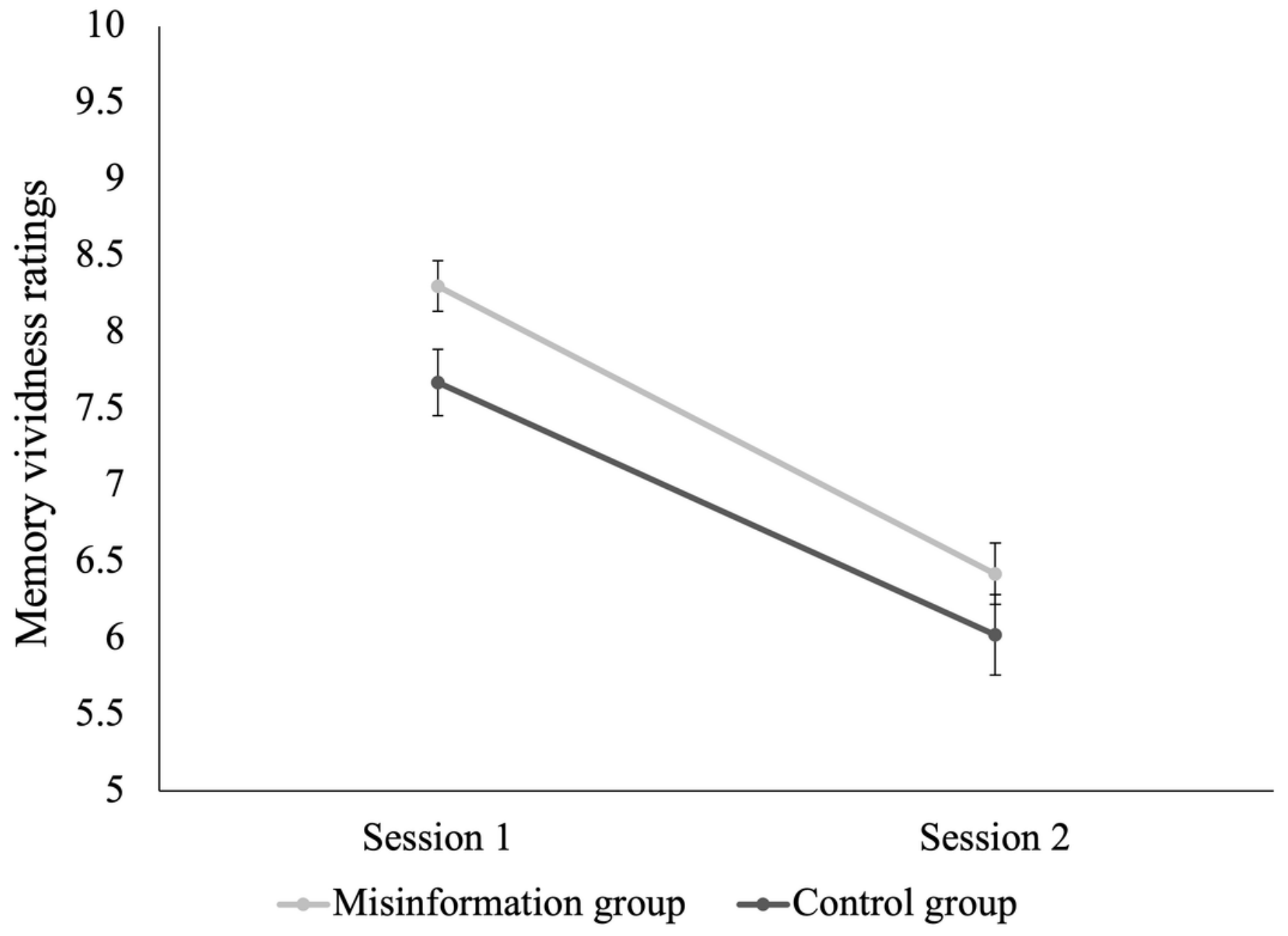

